\title{
Spherical Functions Approach to Sums of Random Hermitian Matrices
}

\author{
Arno B. J. Kuijlaars ${ }^{1}$ and Pablo Román ${ }^{2, *}$ \\ ${ }^{1}$ Department of Mathematics, Katholieke Universiteit Leuven, 3001 \\ Leuven, Belgium and ${ }^{2}$ CIEM, FaMAF, Universidad Nacional de Córdoba, \\ 5000 Córdoba, Argentina
}

*Correspondence to be sent to: e-mail:roman@famaf.unc.edu.ar

We present an approach to sums of random Hermitian matrices via the theory of spherical functions for the Gelfand pair $(\mathrm{U}(n) \ltimes \operatorname{Herm}(n), \mathrm{U}(n))$. It is inspired by a similar approach of Kieburg and Kösters for products of random matrices. The spherical functions have determinantal expressions because of the Harish-Chandra/Itzykson-Zuber integral formula. It leads to remarkably simple expressions for the spherical transform and its inverse. The spherical transform is applied to sums of unitarily invariant random matrices from polynomial ensembles and the subclass of polynomial ensembles of derivative type (in the additive sense), which turns out to be closed under addition. We finally present additional detailed calculations for the sum with a random matrix from a Laguerre unitary ensemble.

\section{Introduction}

There is remarkable recent progress in the understanding of eigenvalues and singular values of products of random matrices. This development started with Akemann and Burda [2] who found explicit formulas for eigenvalues of products of complex Ginibre matrices in terms of Meijer G-functions. It was followed by the works [5, 6], where the same was done for squared singular values of complex Ginibre matrices, and again

Received December 19, 2016; Revised April 3, 2017; Accepted June 1, 2017

Communicated by Prof. Peter Forrester

(C) The Author(s) 2017. Published by Oxford University Press. All rights reserved. For permissions, please e-mail: journals.permission@oup.com. 
the formulas involve Meijer G-functions. Similar expressions were found for eigenvalues and squared singular values for other products of truncated unitary matrices [1, 3], and other random matrices. The results for singular values were subsequently interpreted and extended as transformations of polynomial ensembles in [24-26], see [4] for a survey.

Recently, Kieburg and Kösters [22, 23] presented a natural harmonic analysis point of view on the results on products of random matrices in terms of spherical functions associated with the Gelfand pair $(\mathrm{GL}(n, \mathbb{C}), \mathrm{U}(n))$. It is the goal of this article to give a similar interpretation for sums of random Hermitian matrices in terms of the Gelfand pair $(\mathrm{U}(n) \ltimes \operatorname{Herm}(n), \mathrm{U}(n))$.

In the rest of this section, we summarize our results and state the main theorem. We consider probability density functions $f$ on the space $\operatorname{Herm}(n)$ of $n \times n$ Hermitian matrices that are invariant under conjugation with unitary matrices. Thus $f(X) \mathrm{d} X$ is a probability measure where

$$
\mathrm{d} X=\prod_{j=1}^{n} \mathrm{~d} X_{j j} \prod_{j<k} \mathrm{~d} \operatorname{Re} X_{j, k} d \operatorname{Im} X_{j, k}
$$

is the flat Lebesgue measure on $\operatorname{Herm}(n)$, and we assume

$$
\int f(X) \mathrm{d} X=1, \quad f\left(U X U^{*}\right)=f(X) \geq 0
$$

for every unitary matrix $U \in \mathrm{U}(n)$ and every Hermitian matrix $X \in \operatorname{Herm}(n)$. Then $f$ only depends on the eigenvalues of $X$, say $x_{1}, \ldots, x_{n}$, and we also write $f\left(x_{1}, \ldots, x_{n}\right)$. By the Weyl integration formula we then have that

$$
\frac{\pi^{n(n-1) / 2}}{\prod_{j=1}^{n} j !} f\left(x_{1}, \ldots, x_{n}\right) \Delta_{n}(x)^{2}
$$

is a probability density on $\mathbb{R}^{n}$ where

$$
\Delta_{n}(x)=\prod_{j<k}\left(x_{k}-x_{j}\right)=\operatorname{det}\left[x_{k}^{j-1}\right]_{j, k=1}^{n}
$$

denotes the Vandermonde determinant.

The bounded spherical functions for $(\mathrm{U}(n) \ltimes \operatorname{Herm}(n), \mathrm{U}(n))$ are labelled by $s=\left(s_{1}, \ldots, s_{n}\right) \in \mathbb{R}^{n}$ and are given by

$$
\varphi_{s}(X)=\int_{\mathrm{U}(n)} \mathrm{e}^{i \operatorname{Tr}\left(S U X U^{*}\right)} \mathrm{d} U=\left(\prod_{j=0}^{n-1} j !\right) \frac{\operatorname{det}\left[\mathrm{e}^{i s_{j} x_{k}}\right]_{j, k=1}^{n}}{i^{n(n-1) / 2} \Delta_{n}(s) \Delta_{n}(X)}
$$


where $S=\operatorname{diag}\left(s_{1}, \ldots, s_{n}\right), x=\left(x_{1}, \ldots, x_{n}\right)$ is the vector of eigenvalues of the Hermitian matrix $X$, and $\mathrm{d} U$ is the normalized Haar measure on the unitary group $\mathrm{U}(n)$. This follows from more general results in [8], see also [16] and the discussion in Section 2 below. The second identity in (1.3) is the well-known Harish-Chandra/Itzykson-Zuber formula, see for example [17, Proposition 11.6.1].

The corresponding spherical transform is $f \mapsto \widehat{f}$ where

$$
\widehat{f}(s)=\int f(X) \varphi_{s}(-X) \mathrm{d} X
$$

with the integral over the set of $n \times n$ Hermitian matrices. In terms of an integral over eigenvalues this is by (1.3) and the Weyl integration formula

$$
\widehat{f}(s)=\frac{(\pi i)^{n(n-1) / 2}}{n ! \Delta_{n}(s)} \int_{\mathbb{R}^{n}} f(x) \operatorname{det}\left[\mathrm{e}^{-i s_{j} x_{k}}\right]_{j, k=1}^{n} \Delta_{n}(x) \mathrm{d} x
$$

where $x=\left(x_{1}, \ldots, x_{n}\right)$ and $\mathrm{d} x=\mathrm{d} x_{1} \cdots d x_{n}$. The prefactor in (1.5) is such that

$$
\widehat{f}(0, \ldots, 0)=\int f(X) \mathrm{d} X=1,
$$

which should hold since $f$ is a probability density function and $\varphi_{(0, \ldots, 0)}(X)=1$ for every $X \in \operatorname{Herm}(n)$.

In Section 2 below we show that there is an inversion formula

$$
f(x)=\frac{(\pi i)^{-n(n-1) / 2}}{(2 \pi)^{n} n ! \Delta_{n}(x)} \int_{\mathbb{R}^{n}} \widehat{f}(s) \operatorname{det}\left[\mathrm{e}^{i s_{j} x_{k}}\right]_{j, k=1}^{n} \Delta_{n}(s) \mathrm{d} s,
$$

which basically follows from the multidimensional inverse Fourier transform.

Our main result is the following.

Theorem 1.1. Suppose that $X$ and $Y$ are independent unitarily invariant random Hermitian matrices with probability densities $f_{X}$ and $f_{Y}$, respectively. Let $f_{X+Y}$ be the probability density of the sum $X+Y$. Then

$$
\widehat{f}_{X+Y}=\widehat{f}_{X} \cdot \widehat{f}_{Y}
$$

and

$$
f_{X+Y}(x)=\frac{(\pi i)^{-n(n-1) / 2}}{(2 \pi)^{n} n ! \Delta_{n}(x)} \int_{\mathbb{R}^{n}} \widehat{f}_{X}(s) \widehat{f}_{Y}(s) \operatorname{det}\left[\mathrm{e}^{i s_{j} x_{k}}\right]_{j, k=1}^{n} \Delta_{n}(s) \mathrm{d} s
$$


The formula (1.8) is of course the inversion formula (1.6) applied to (1.7).

The formulas (1.7) and (1.8) are analogues of familiar properties of the usual Fourier transform. We emphasize that they hold for random unitarily invariant Hermitian matrices. The situation for real orthogonally invariant symmetric matrices is not so nice, because of the lack of a Harish-Chandra/Itzykson-Zuber formula (1.3) for the corresponding integral over the orthogonal group.

The proof of Theorem 1.1 is in Section 2. We discuss the Gelfand pair $(\mathrm{U}(n) \ltimes$ $\operatorname{Herm}(n), \mathrm{U}(n))$ and its relevance for sums of random Hermitian matrices. Then we recall the general concept of a Gelfand pair, the notion of spherical functions and spherical transform. Then we specialize again to the case $(\mathrm{U}(n) \ltimes \operatorname{Herm}(n), \mathrm{U}(n))$ and we show that the bounded spherical functions are given by the functions (1.3). We compute the spherical transform (1.5) and its inverse (1.6) and then finish the proof of Theorem 1.1.

In Section 3, we compute the spherical transform $\widehat{f}$ in certain situations. The result is explicit for the probability density functions of the Gaussian unitary ensemble (GUE) and Laguerre unitary ensemble (LUE) random matrix ensembles. For a polynomial ensemble, the spherical transform is a ratio of determinants (3.11) with a Vandermonde determinant in the denominator. From Theorem 1.1, we then find that the sum of a polynomial ensemble with a GUE or LUE matrix is again a polynomial ensemble, see Corollaries 3.1 and 3.2. The result for GUE was already noted in [12], while it is a new result for the sum with an LUE matrix, although special cases are contained in [18, 27]. We end Section 3 with polynomial ensembles of derivative type, following the similar notion introduced in $[22,23]$ in a multiplicative setting.

In Section 4, we provide more information on the sum of a polynomial ensemble with an LUE matrix. We give a second proof of Corollary 3.2, as well as transformation results for the correlation kernel and the biorthogonal functions that are associated with a polynomial ensemble.

Remark 1.2. The spherical functions for the Gelfand pair $(\mathrm{GL}(n, \mathbb{C}), \mathrm{U}(n))$ can be written as

$$
\phi_{s}(X)=\left(\prod_{j=0}^{n-1} j !\right) \frac{\operatorname{det}\left[x_{j}^{s_{k}}\right]_{j, k=1}^{n}}{\Delta_{n}(s) \Delta_{n}(x)}, \quad X \in \mathrm{GL}(n, \mathbb{C}),
$$

where $x_{1}, \ldots, x_{n}$ are the eigenvalues of $X^{*} X$, see $[19,23]$. These functions are used in [23] for products of random matrices, in a similar way as (1.3) will be used for sums of random matrices in this article. 
Remark 1.3. Let $G$ be a connected semisimple Lie group with finite centre and let $K$ be a maximal compact subgroup so that $G / K$ is a Riemannian symmetric space of the noncompact type. Let $\mathfrak{g}=\mathfrak{k} \oplus \mathfrak{p}$ be a Cartan decomposition of the Lie algebra of $G$. In [7, Theorem 3.4] it is shown that the spherical functions for the flat symmetric space $G_{0} / K$, where $G_{0}=K \ltimes \mathfrak{p}$, can be obtained as limits of the spherical functions for $G / K$. A related analysis can be found in [20, Chapter IV, Proposition 4.10], where the spherical functions for $G / K$, with $G$ complex, are given in terms of the spherical functions for $G_{0} / K$.

In our setting, it turns out that the explicit expression (1.3) can be alternatively obtained from the limit

$$
\varphi_{s}(X)=\lim _{\varepsilon \rightarrow 0} \phi_{\frac{i s}{\epsilon}}\left(\mathrm{e}^{\frac{\epsilon X}{2}}\right), \quad X \in \operatorname{Herm}(n),
$$

where $\phi_{s}$ are the spherical functions for $(\mathrm{GL}(n, \mathbb{C}), \mathrm{U}(n))$ as in Remark 1.2.

\section{Spherical Functions}

\subsection{The Gelfand pair}

The Gelfand pair $(\mathrm{U}(n) \ltimes \operatorname{Herm}(n), \mathrm{U}(n))$ consists of the semidirect product $G=\mathrm{U}(n) \ltimes$ $\operatorname{Herm}(n)$ of the unitary group with the real vector space $\operatorname{Herm}(n)$ of complex Hermitian $n \times n$ matrices and the compact subgroup $K=\mathrm{U}(n) \times\{0\} \simeq \mathrm{U}(n)$. A unitary matrix $U \in \mathrm{U}(n)$ acts on $\operatorname{Herm}(n)$ by conjugation $A \mapsto U A U^{*}$. The composition law on $G$ is

$$
\left(U_{1}, A\right) \cdot\left(U_{2}, B\right)=\left(U_{1} U_{2}, A+U_{1} B U_{1}^{*}\right)
$$

for $U_{1}, U_{2} \in \mathrm{U}(n)$ and $A, B \in \operatorname{Herm}(n)$.

A function $f: G \rightarrow \mathbb{C}$ is bi-K-invariant if it is invariant under left and right multiplication with elements of $K$. In our situation it means that $f(U, A)$ only depends on the eigenvalues of the Hermitian matrix $A$. For a bi- $K$-invariant function $f$ we therefore simply write $f(A)$ instead of $f(U, A)$, or even $f\left(x_{1}, \ldots, x_{n}\right)$ where $x_{1}, \ldots, x_{n}$ are the eigenvalues of $A$, see also the introduction.

There is a convolution product for functions on $G$, which for bi- $K$-invariant functions reduces to (it is a simple verification)

$$
(f * g)(A)=\int_{\operatorname{Herm}(n)} f(X) g(A-X) \mathrm{d} X
$$

where $\mathrm{d} X$ is the Lebesgue measure on $\operatorname{Herm}(n)$, see (1.1). From (2.2) it is obvious that $f * g=g * f$ for bi- $K$-invariant functions, which is the property that defines a Gelfand pair $(G, K)$. 
6 A. B. J. Kuijlaars et al.

The connection to random matrices is in the following corollary.

Corollary 2.1. If $f_{X}$ and $f_{Y}$ are the probability densities for independent bi- $K$-invariant random Hermitian matrices $X$ and $Y$ then $f_{X} * f_{Y}=f_{X+Y}$ is the probability density for the $\operatorname{sum} X+Y$.

\subsection{Spherical functions: general concepts}

We follow the exposition of van Dijk [14].

In this subsection we let $G$ be a general locally compact group with a compact subgroup $K$. Later we will specialize it to the case $G=\mathrm{U}(n) \ltimes \operatorname{Herm}(n)$ and $K=\mathrm{U}(n)$. In the general setting we use lower case letters for elements of $G$ and $K$. Let $d x$ be left Haar measure on $G$ and let $d k$ be normalized Haar measure on $K$.

Let $C_{c}^{\#}(G)$ be the space of continuous compactly supported complex valued functions on $G$ that are bi-invariant with respect to $K$. The group structure on $G$ gives rise to a convolution product

$$
(f * g)(x)=\int_{G} f(y) g\left(y^{-1} x\right) \mathrm{d} y
$$

for functions $f$ and $g$ on $G$. When restricted to $C_{c}^{\#}(G)$ it turns $C_{c}^{\#}(G)$ into a convolution algebra. The pair $(G, K)$ is called a Gelfand pair if $C_{C}^{\#}(G)$ is commutative.

A spherical function for a Gelfand pair $(G, K)$ is a nonzero continuous function $\varphi$ on $G$ such that

$$
\int_{K} \varphi(x k y) \mathrm{d} k=\varphi(x) \varphi(y), \quad x, y \in G
$$

It is equivalent to saying that the functional $\chi$ on $C_{c}^{\#}(G)$ defined by

$$
\chi(f)=\int_{G} f(x) \varphi\left(x^{-1}\right) \mathrm{d} x
$$

is a non-trivial character, that is,

$$
\chi(f * g)=\chi(f) \chi(g),
$$

see [14, Proposition 6.1.5]. A spherical function $\varphi$ is bi- $K$-invariant and $\varphi(e)=1$, where $e$ is the unit element in $G$.

A locally integrable function $\varphi: G \rightarrow \mathbb{C}$ is positive-definite if

$$
\int_{G} \int_{G} \varphi\left(x^{-1} y\right) f(x) \overline{f(y)} \mathrm{d} x \mathrm{~d} y \geq 0
$$


for every continuous function $f$ with compact support on $G$. If $\pi$ is a unitary representation of $G$ on a Hilbert space $H$, and $\varepsilon \in H$, then $X \mapsto\langle\varepsilon, \pi(x) \varepsilon\rangle$ is a bounded continuous positive-definite function on $G$, and every bounded continuous positive-definite function is obtained this way. We may assume in addition that $\varepsilon$ is a cyclic vector, see [14, Remark 5.1.7].

A continuous positive-definite function $\varphi$ on $G$ that is bi- $K$-invariant with $\varphi(e)=$ 1 is a spherical function if and only if the associated unitary representation is irreducible [14, Theorems 5.3.2 and 6.2.5].

Let $Z$ be the set of positive-definite spherical functions. Such functions are automatically continuous and bounded. Let $L^{1}(G)^{\#}$ denote the subspace of bi- $K$-invariant functions in $L^{1}(G)$. Then the spherical transform $\widehat{f}$ of a function $f \in L^{1}(G)^{\#}$ is defined as

$$
\widehat{f}: Z \rightarrow \mathbb{C}: \quad \varphi \in Z \mapsto \widehat{f}(\varphi)=\int_{G} f(x) \varphi\left(x^{-1}\right) \mathrm{d} x
$$

see [14, Definition 6.4.3] where it is called the Fourier transform. There is a natural topology on $Z$, which is locally compact. Then by [14, page 84$], \widehat{f}$ is a continuous function on $Z$ that vanishes at infinity, and

$$
|\widehat{f}(\varphi)| \leq \int_{G}|f(x)| \mathrm{d} x, \quad \varphi \in Z,
$$

the transformation $f \mapsto \widehat{f}$ is linear with

$$
\widehat{f * g}=\widehat{f} \widehat{g}, \quad f, g \in L^{1}(G)^{\#}
$$

There is an inversion formula according to which we can recover $f$ from $\widehat{f}$. Namely, there is a unique measure $v$ on $Z$ (sometimes called Plancherel measure) such that for bi- $K$-invariant functions $f$,

$$
f(x)=\int_{Z} \varphi(x) \widehat{f}(\varphi) \mathrm{d} \nu(\varphi)
$$

and there is a Plancherel formula

$$
\int_{G}|f(x)|^{2} \mathrm{~d} x=\int_{Z}|\widehat{f}(\varphi)|^{2} \mathrm{~d} v(\varphi), \quad f \in L^{2}(G)^{\#}
$$

see [14, Theorems 6.4.5 and 6.4.6]. 
8 A. B. J. Kuijlaars et al.

\subsection{Spherical functions in special case}

Suppose the Gelfand pair takes the form $(K \ltimes N, K)$ where $K$ is a compact group that acts on $N$. Then bi- $K$-invariant functions on $G=K \ltimes N$ are naturally identified with functions on $N$ that are invariant under the action of $K$. This situation was considered by Benson et al. [8]. When $N$ is a nilpotent Lie group, they characterized the spherical functions as follows.

Lemma 2.2. [8, Lemma 8.2 and Corollary 8.4] Suppose $\varphi$ is a bounded spherical function on $N$, where $N$ is a nilpotent Lie group. Then $\varphi$ is positive-definite, and there exist an irreducible unitary representation $\pi$ of $N$ on a Hilbert space $H_{\pi}$ and a unit vector $\xi \in H_{\pi}$ such that

$$
\varphi(x)=\int_{K}\langle\pi(k \cdot x) \xi, \xi\rangle \mathrm{d} k
$$

for each $x \in N$.

Lemma 2.2 applies to the Gelfand pair $(\mathrm{U}(n) \ltimes \operatorname{Herm}(n), \mathrm{U}(n))$ since the vector space $\operatorname{Herm}(n)$ is an abelian group, and thus nilpotent. The pairing $\langle A, B\rangle=\operatorname{Tr}(A B)$ is a real inner product on $\operatorname{Herm}(n)$, and all irreducible unitary representations of $\operatorname{Herm}(n)$ are given by $\pi_{S}: \operatorname{Herm}(n) \rightarrow \mathbb{C}$, where

$$
\pi_{S}: X \mapsto \mathrm{e}^{i\langle S, X\rangle}=\mathrm{e}^{i \operatorname{Tr}(S X)}, \quad S \in \operatorname{Herm}(n)
$$

From Lemma 2.2 we thus obtain that all bounded positive-definite spherical functions are given by

$$
\begin{aligned}
\varphi_{S}(A) & =\int_{\mathrm{U}(n)} \pi_{S}\left(U A U^{*}\right) \mathrm{d} U \\
& =\int_{\mathrm{U}(n)} \mathrm{e}^{i \operatorname{Tr}\left(S U A U^{*}\right)} \mathrm{d} U, \quad A \in \operatorname{Herm}(n),
\end{aligned}
$$

where $\mathrm{d} U$ denotes the normalized Haar measure on $\mathrm{U}(n)$, and $S \in \operatorname{Herm}(n)$.

Remark 2.3. In fact we can verify the property (2.3) directly from (2.7), for any fixed $n \times n$ matrix $S$. If $x=\left(U_{1}, A\right) \in G, k=(V, 0) \in K$ and $y=\left(U_{2}, B\right) \in G$, then by the composition rule (2.1) we have

$$
x k y=\left(U_{1} V U_{2}, A+U_{1} V B V^{*} U_{1}^{*}\right) .
$$


The definition (2.7) only uses the Hermitian part of $x k y$ and we have for Hermitian matrices $A$ and $B$,

$$
\begin{aligned}
\int_{\mathrm{U}(n)} \varphi_{S}\left(A+U_{1} V B V^{*} U_{1}^{*}\right) \mathrm{d} V & =\int_{\mathrm{U}(n)} \int_{\mathrm{U}(n)} \mathrm{e}^{i \operatorname{Tr}\left(S U\left(A+U_{1} V B V^{*} U_{1}^{*}\right) U^{*}\right)} \mathrm{d} U \mathrm{~d} V \\
& =\int_{\mathrm{U}(n)} \mathrm{e}^{i \operatorname{Tr} S U A U^{*}} \int_{\mathrm{U}(n)} \mathrm{e}^{i \operatorname{Tr} S U U_{1} V B V^{*} U_{1}^{*} U^{*}} \mathrm{~d} V \mathrm{~d} U,
\end{aligned}
$$

where we used the linearity of the trace. Changing $V \mapsto U_{1}^{*} U^{*} V$ in the integral in (2.8), using the invariance of Haar measure, and recalling (2.7), we find indeed

$$
\int_{\mathrm{U}(n)} \int_{\mathrm{U}(n)} \mathrm{e}^{i \operatorname{Tr}\left(S U\left(A+U_{1} V B V^{*} U_{1}^{*}\right) U^{*}\right)} \mathrm{d} U \mathrm{~d} V=\varphi_{S}(A) \varphi_{S}(B) .
$$

It is also clear from (2.7) that $\varphi_{S}(0)=1$ and thus $\varphi_{S}$ is a spherical function. It is bounded if and only if $S \in \operatorname{Herm}(n)$.

For every matrix $S \in \operatorname{Herm}(n)$ there exists $U \in \mathrm{U}(n)$ such that $U S U^{*}=$ $\operatorname{diag}\left(s_{1}, \ldots, s_{n}\right)$ where $s_{j} \in \mathbb{R}$ for $j=1, \ldots, n$. By (2.7) and the invariance of the Haar measure, the spherical function only depends on the eigenvalues of $S$ and we write $\varphi_{s}$ instead of $\varphi_{S}$ where $s=\left(s_{1}, \ldots, s_{n}\right)$. Moreover, $\varphi_{\left(s_{1}, \ldots, s_{n}\right)}=\varphi_{\left(s_{\sigma(1)}, \ldots, s_{\sigma(n)}\right)}$ for any permutation $\sigma \in S_{n}$. Therefore the set $Z$ of positive-definite spherical functions can be identified with $\mathbb{R}^{n} / S_{n}$.

By the Harish-Chandra/Itzykson-Zuber formula the spherical function (2.7) takes the determinantal form (1.3). The formula should be understood in a limiting sense if some of the $x_{j}{ }^{\prime}$ s and/or some of the $s_{j}$ 's coincide.

\subsection{Proof of Theorem 1.1}

The spherical transform (2.4) of a function $f \in L^{1}(G)^{\#}$ is in our special case $(G, K)=$ $(\mathrm{U}(n) \ltimes \operatorname{Herm}(n), \mathrm{U}(n))$,

$$
\widehat{f}(s)=\int_{\operatorname{Herm}(n)} f(A) \varphi_{s}(-A) \mathrm{d} A,
$$

which we view as a function on $\mathbb{R}^{n}$ that is invariant under permutation of coordinates (instead of a function on $Z=\mathbb{R}^{n} / S_{n}$ ). If $f$ has compact support, then (2.9) is defined for all $s \in \mathbb{C}^{n}$. As an integral over the eigenvalues, the spherical transform (2.9) gives us (1.5) by the Weyl integration formula. Then (1.7) in Theorem 1.1 follows because of Corollary 2.1 and (2.5). 
10 A. B. J. Kuijlaars et al.

Next, we expand the determinant $\operatorname{det}\left[\mathrm{e}^{-i s_{j} x_{k}}\right]_{j, k=1}^{n}$ to obtain from (1.5) that

$$
\widehat{f}(s) \Delta_{n}(s)=\frac{(\pi i)^{n(n-1) / 2}}{n !} \sum_{\sigma \in S_{n}} \operatorname{sgn}(\sigma) \int_{\mathbb{R}^{n}} f(x)\left[\prod_{j=1}^{n} \mathrm{e}^{-i s_{j} x_{\sigma(j)}}\right] \Delta_{n}(x) \mathrm{d} x,
$$

where the sum is over permutations $\sigma \in S_{n}$. Because $f(x)=f\left(x_{1}, \ldots, x_{n}\right)$ is invariant under permutations of coordinates, while $\Delta_{n}(x)$ changes sign for odd permutations, each permutation $\sigma$ has the same contribution. Thus

$$
\widehat{f}(s) \Delta_{n}(s)=(\pi i)^{n(n-1) / 2} \int_{\mathbb{R}^{n}} f(x)\left[\prod_{j=1}^{n} \mathrm{e}^{-i s_{j} x_{j}}\right] \Delta_{n}(x) \mathrm{d} x
$$

which is the usual $n$-dimensional Fourier transform of $f(x) \Delta_{n}(x)$. Thus by Fourier inversion

$$
f(x) \Delta_{n}(x)=\frac{1}{(2 \pi)^{n}(\pi i)^{n(n-1) / 2}} \int_{\mathbb{R}^{n}} \widehat{f}(s)\left[\prod_{j=1}^{n} \mathrm{e}^{i s_{j} x_{j}}\right] \Delta_{n}(s) \mathrm{d} s
$$

with $\mathrm{d} s=\mathrm{d} s_{1} \cdots \mathrm{d} s_{n}$. Now $\widehat{f}(s)$ is invariant under permutations of $s_{1}, \ldots, s_{n}$. Then by similar argument as above, we can write

$$
\begin{aligned}
f(x) \Delta_{n}(x) & =\frac{1}{(2 \pi)^{n}(\pi i)^{n(n-1) / 2} n !} \sum_{\sigma \in S_{n}} \operatorname{sgn}(\sigma) \int_{\mathbb{R}^{n}} \widehat{f}(s)\left[\prod_{j=1}^{n} \mathrm{e}^{i s_{\sigma(j)} x_{j}}\right] \Delta_{n}(s) \mathrm{d} s \\
& =\frac{1}{(2 \pi)^{n}(\pi i)^{n(n-1) / 2} n !} \int_{\mathbb{R}^{n}} \widehat{f}(s) \operatorname{det}\left[\mathrm{e}^{i s_{k} x_{j}}\right]_{j, k=1}^{n} \Delta_{n}(s) \mathrm{d} s
\end{aligned}
$$

which is (1.6). This proves the inversion formula (1.8) and the proof of Theorem 1.1 is complete.

Remark 2.4. Recalling the expression (1.3) of the spherical function, and writing $f(A)$ instead of $f(x)$, we have

$$
f(A)=\frac{1}{(2 \pi)^{n} \pi^{n(n-1) / 2} \prod_{j=0}^{n} j !} \int_{\mathbb{R}^{n}} \widehat{f}(s) \varphi_{s}(A) \Delta_{n}(s)^{2} \mathrm{~d} s .
$$

So the Plancherel measure on $Z$ is proportional to $\Delta_{n}(s)^{2} d s$. 


\section{Computation of Special Cases}

The integral (1.5) can be evaluated explicitly in certain cases. The Andreief formula [13]

$$
\int \operatorname{det}\left[f_{j}\left(x_{k}\right)\right]_{j, k=1}^{n} \operatorname{det}\left[g_{j}\left(x_{k}\right)\right]_{j, k=1}^{n} \mathrm{~d} x_{1} \cdots \mathrm{d} x_{n}=n ! \operatorname{det}\left[\int f_{j}(x) g_{k}(x) \mathrm{d} x\right]_{j, k=1}^{n}
$$

will be useful in the computations.

\subsection{Gaussian unitary ensemble}

The density of the GUE is

$$
f_{G U E}(X)=\frac{1}{2^{n / 2} \pi^{n^{2} / 2}} \mathrm{e}^{-\frac{1}{2} \operatorname{Tr} X^{2}} .
$$

In terms of eigenvalues we have

$$
f_{G U E}\left(x_{1}, \ldots, x_{n}\right)=\frac{1}{2^{n / 2} \pi^{n^{2} / 2}} \prod_{k=1}^{n} \mathrm{e}^{-\frac{1}{2} x_{k}^{2}}
$$

and then by (1.5) and the Andreief formula (3.1),

$$
\widehat{f}_{G U E}(s)=\frac{i^{n(n-1) / 2}}{(2 \pi)^{n / 2} \Delta_{n}(s)} \operatorname{det}\left[\int_{-\infty}^{\infty} \mathrm{e}^{-\frac{1}{2} x^{2}} \mathrm{e}^{-i s_{j} x} x^{k-1} \mathrm{~d} x\right]_{j, k=1}^{n} .
$$

The integrals can be evaluated, since

$$
\frac{1}{\sqrt{2 \pi}} \int_{-\infty}^{\infty} \mathrm{e}^{-\frac{1}{2} x^{2}} \mathrm{e}^{-i s x} \mathrm{dx}=\mathrm{e}^{-\frac{1}{2} s^{2}}
$$

and for $k=2, \ldots, n$,

$$
\frac{1}{\sqrt{2 \pi}} \int_{-\infty}^{\infty} \mathrm{e}^{-\frac{1}{2} x^{2}} \mathrm{e}^{-i s x} s^{k-1} \mathrm{~d} x=\left(i \frac{\mathrm{d}}{\mathrm{d} s}\right)^{k-1}\left(\mathrm{e}^{-\frac{1}{2} s^{2}}\right)=(-i)^{k-1} P_{k-1}(s) \mathrm{e}^{-\frac{1}{2} s^{2}}
$$

for a certain monic polynomial $P_{k-1}$ of degree $k-1$ (which is actually a multiple of the usual Hermite polynomial). Inserting this into (3.3) we obtain

$$
\begin{aligned}
\widehat{f}_{G U E}(s) & =\frac{i^{n(n-1) / 2}}{\Delta_{n}(s)} \operatorname{det}\left[(-i)^{k-1} P_{k-1}\left(s_{j}\right) \mathrm{e}^{-\frac{1}{2} s_{j}^{2}}\right]_{j, k=1}^{n} \\
& =\frac{i^{n(n-1) / 2}}{\Delta_{n}(s)}\left(\prod_{k=1}^{n}(-i)^{k-1}\right) \operatorname{det}\left[P_{k-1}\left(s_{j}\right)\right]_{j, k=1}^{n}\left(\prod_{j=1}^{n} \mathrm{e}^{-\frac{1}{2} s_{j}^{2}}\right) \\
& =\prod_{j=1}^{n} \mathrm{e}^{-\frac{1}{2} s_{j}^{2}}
\end{aligned}
$$


12 A. B. J. Kuijlaars et al.

where we used the fact that for any sequence of monic polynomials $\left(P_{j}\right)_{j=0}^{n-1}$ with $\operatorname{deg} P_{j}=j$, one has $\operatorname{det}\left[P_{k-1}\left(s_{j}\right)\right]_{j, k=1}^{n}=\Delta_{n}(s)$.

\subsection{Laguerre unitary ensemble}

The LUE with parameter $\alpha>-1$ is given by the probability density

$$
f_{L U E}(L)=\frac{(\operatorname{det} L)^{\alpha}}{\pi^{n(n-1) / 2} \prod_{j=1}^{n} \Gamma(\alpha+j)} \mathrm{e}^{-\operatorname{Tr} L}
$$

on the set of positive definite Hermitian matrices $L$. Thus

$$
f_{L U E}\left(x_{1}, \ldots, x_{n}\right)=\frac{1}{\pi^{n(n-1) / 2} \prod_{j=1}^{n} \Gamma(\alpha+j)} \prod_{k=1}^{n} x_{k}^{\alpha} \mathrm{e}^{-x_{k}} \mathbf{1}_{x_{k} \geq 0} .
$$

By (1.5) and the Andreief formula (3.1),

$$
\widehat{f}_{L U E}(s)=\frac{i^{n(n-1) / 2}}{\prod_{j=1}^{n} \Gamma(\alpha+j) \Delta_{n}(s)} \operatorname{det}\left[\int_{0}^{\infty} x^{\alpha+k-1} \mathrm{e}^{-x} \mathrm{e}^{-i s_{j} x} \mathrm{~d} x\right]_{j, k=1}^{n} .
$$

We compute the integrals

$$
\int_{0}^{\infty} x^{\alpha+k-1} \mathrm{e}^{-x} \mathrm{e}^{-i s x} \mathrm{~d} x=\frac{\Gamma(\alpha+k)}{(1+i s)^{\alpha+k}}
$$

and (3.6) simplifies to

$$
\begin{aligned}
\widehat{f}_{L U E}(s) & =\frac{i^{n(n-1) / 2}}{\Delta_{n}(s)} \operatorname{det}\left[\frac{1}{\left(1+i s_{j}\right)^{\alpha+k}}\right]_{j, k=1}^{n} \\
& =\frac{i^{n(n-1) / 2}}{\Delta_{n}(s)} \operatorname{det}\left[\left(1+i s_{j}\right)^{n-k}\right]_{j, k=1}^{n} \prod_{j=1}^{n} \frac{1}{\left(1+i s_{j}\right)^{\alpha+n}} .
\end{aligned}
$$

The remaining determinant in (3.7) is $(-i)^{n(n-1) / 2} \Delta_{n}(s)$ and we find the following spherical transform for the LUE density

$$
\widehat{f}_{L U E}(s)=\prod_{j=1}^{n} \frac{1}{\left(1+i s_{j}\right)^{\alpha+n}} .
$$

\subsection{Polynomial ensemble}

A polynomial ensemble $[25,26]$ is a probability density on $\mathbb{R}^{n}$ of the form

$$
\frac{1}{Z_{n}} \Delta_{n}(x) \operatorname{det}\left[w_{k}\left(x_{j}\right)\right]_{j, k=1}^{n}
$$


for some given functions $w_{1}, \ldots, w_{n}$, and a certain normalization constant $Z_{n}$. If $X$ is a random Hermitian matrix, then we write

$$
X \sim P E\left(w_{1}, \ldots, w_{n}\right)
$$

if the induced probability density on the eigenvalues is of the form (3.9). If $X$ is unitarily invariant with a probability density $f$, then in view of (1.2) this means that

$$
f(A)=f\left(x_{1}, \ldots, x_{n}\right)=\frac{1}{Z_{n}^{\prime}} \frac{\operatorname{det}\left[w_{k}\left(x_{j}\right)\right]_{j, k=1}^{n}}{\Delta_{n}(x)} .
$$

Using (3.10) in (1.5) we find for the spherical transform of a polynomial ensemble

$$
\widehat{f}\left(s_{1}, \ldots, s_{n}\right)=\frac{1}{Z_{n}^{\prime \prime} \Delta_{n}(s)} \operatorname{det}\left[\int_{-\infty}^{\infty} w_{k}(x) \mathrm{e}^{-i s_{j} x} \mathrm{~d} x\right]_{j, k=1}^{n},
$$

where we also used the Andreief formula (3.1). The normalization constant is such that $\widehat{f}(0, \ldots, 0)=1$, which by l'Hopital's rule means that

$$
Z_{n}^{\prime \prime}=\frac{(-i)^{n(n-1) / 2}}{\prod_{j=0}^{n-1} j !} \operatorname{det}\left[\int_{-\infty}^{\infty} w_{k}(x) x^{j-1} \mathrm{~d} x\right]_{j, k=1}^{n} .
$$

We now recover the following result that was proved in a different way by Claeys et al. [12, Theorem 2.1].

Corollary 3.1. Let $X \sim P E\left(f_{1}, \ldots, f_{n}\right)$ be an $n \times n$ unitarily invariant random Hermitian matrix for certain functions $f_{1}, \ldots, f_{n}$. Let $Y$ be an $n \times n$ GUE matrix, independent of $X$. Then $X+Y \sim P E\left(g_{1}, \ldots, g_{n}\right)$ where

$$
g_{k}(y)=\int_{-\infty}^{\infty} \mathrm{e}^{-\frac{1}{2} x^{2}} f_{k}(y-x) \mathrm{d} x
$$

Proof. We have by (3.11)

$$
\widehat{f}_{X}(s) \propto \frac{1}{\Delta_{n}(s)} \operatorname{det}\left[\int_{-\infty}^{\infty} f_{k}(x) \mathrm{e}^{-i s_{j} x} \mathrm{~d} x\right]_{j, k=1}^{n}
$$

and by (3.4)

$$
\widehat{f}_{Y}(s)=\prod_{j=1}^{n} \mathrm{e}^{-\frac{1}{2} s_{j}^{2}}
$$


14 A. B. J. Kuijlaars et al.

Then by Theorem 1.1

$$
\begin{aligned}
\widehat{f}_{X+Y}(s) & \propto \frac{1}{\Delta_{n}(s)} \operatorname{det}\left[\int_{-\infty}^{\infty} f_{k}(x) \mathrm{e}^{-i s_{j} x} \mathrm{~d} x\right]_{j, k=1}^{n} \prod_{j=1}^{n} \mathrm{e}^{-\frac{1}{2} s_{j}^{2}} \\
& =\frac{1}{\Delta_{n}(s)} \operatorname{det}\left[\mathrm{e}^{-\frac{1}{2} s_{j}^{2}} \int_{-\infty}^{\infty} f_{k}(x) \mathrm{e}^{-i s_{j} x} \mathrm{~d} x\right]_{j, k=1}^{n} \\
& \propto \frac{1}{\Delta_{n}(s)} \operatorname{det}\left[\left(\mathcal{F}\left[\mathrm{e}^{-\frac{1}{2} x^{2}}\right]\right)\left(s_{j}\right)\left(\mathcal{F} f_{k}\right)\left(s_{j}\right)\right]_{j, k=1}^{n}
\end{aligned}
$$

where $\mathcal{F}$ is the Fourier transform

$$
(\mathcal{F} W)(s)=\int_{-\infty}^{\infty} w(x) \mathrm{e}^{-i s x} \mathrm{~d} x
$$

The function $g_{k}$ from (3.12) is the convolution of $f_{k}$ with $x \mapsto \mathrm{e}^{-\frac{1}{2} x^{2}}$ and by elementary properties of the Fourier transform it follows from (3.13) that

$$
\widehat{f}_{X+Y}(s) \propto \frac{1}{\Delta_{n}(s)} \operatorname{det}\left[\mathcal{F} g_{k}\left(s_{j}\right)\right]_{j, k=1}^{n}
$$

which by (3.11) and the injectivity of the spherical transform means that $X+Y \sim$ $P E\left(g_{1}, \ldots, g_{n}\right)$ as claimed.

In the same way we combine (3.11) and (3.8) and find the following new result about addition of an LUE matrix.

Corollary 3.2. Let $X$ be an $n \times n$ unitarily invariant random Hermitian matrix such that $X \sim P E\left(f_{1}, \ldots, f_{n}\right)$ for certain functions $f_{1}, \ldots, f_{n}$. Let $L$ be an $n \times n$ LUE matrix with parameter $\alpha$, independent of $X$. Then $X+L \sim P E\left(g_{1}, \ldots, g_{n}\right)$ where

$$
g_{k}(y)=\int_{0}^{\infty} x^{\alpha+n-1} \mathrm{e}^{-x} f_{k}(y-x) \mathrm{d} x
$$

Proof. The proof is exactly the same as the proof of Corollary 3.1. We only use (3.8) instead of (3.4) and the fact that

$$
\mathcal{F}\left[x^{\alpha+n-1} \mathrm{e}^{-x} \mathbf{1}_{x \geq 0}\right](s)=\frac{\Gamma(\alpha+n)}{(1+i s)^{n}},
$$

where $\mathcal{F}$ is the Fourier transform as in (3.14). 
There is an interesting alternative proof of Corollary 3.2, along the lines of the proof in [12] of Corollary 3.1, which we give in Section 4 .

Remark 3.3. We note that Forrester and Rains [18, appendix E] and Kumar [27, 28] obtain the biorthogonal determinantal structure for a number of sums of random matrices, including the sum of an LUE and a GUE matrix and the sum of two LUE matrices.

In [27], Kumar considers sums $A A^{*}+B B^{*}$ where $A$ and $B$ are independent complex Wishart matrices of sizes $n \times\left(n+v_{A}\right)$ and $n \times\left(n+v_{B}\right)$, respectively, and with covariance matrices $\Sigma_{A}$ and $\Sigma_{B}$. In case one of the covariance matrices is a multiple of the identity, then [27, formula (15)] gives an explicit formula for the joint densities of the eigenvalues of $A A^{*}+B B^{*}$ as a polynomial ensemble.

The formula can be obtained from Corollary 3.2. Indeed, let us take $\Sigma_{A}=I$, and the eigenvalues of $\Sigma_{B}$ are $\sigma_{1}, \ldots, \sigma_{n}$, which for simplicity we assume to be all distinct. Then $L=A A^{*}$ is an LUE matrix with parameter $v_{A}$ and the eigenvalues of $X=B B^{*}$ have the joint density

$$
\propto \Delta_{n}(x) \operatorname{det}\left[x_{j}^{v_{B}} \mathrm{e}^{-\frac{x_{j}}{\sigma_{k}}}\right]_{j, k=1=1}^{n}, \quad \text { all } x_{j}>0,
$$

that is, $X \sim P E\left(f_{1}, \ldots, f_{n}\right)$ with $f_{k}(x)=X^{v_{B}} \mathrm{e}^{-\frac{X}{\sigma_{k}}} \mathbf{1}_{x \geq 0}$ for $k=1, \ldots, n$. Then by Corollary 3.2 $L+X \sim P E\left(g_{1}, \ldots, g_{n}\right)$ with

$$
g_{k}(y)=\int_{0}^{\infty} x^{v_{A}+n-1} \mathrm{e}^{-x} f_{k}(y-x) \mathrm{d} x=\int_{0}^{y} x^{v_{A}+n-1} \mathrm{e}^{-x}(y-x)^{v_{B}} \mathrm{e}^{-\frac{Y-X}{\sigma_{k}}} \mathrm{~d} x
$$

The change of variables $x=y(1-s)$ then gives

$$
\begin{aligned}
g_{k}(y) & =y^{v_{A}+v_{B}+n} \mathrm{e}^{-Y} \int_{0}^{1} s^{v_{B}}(1-s)^{v_{A}+n-1} \mathrm{e}^{\left(1-\sigma_{k}^{-1}\right) Y s} \mathrm{~d} s \\
& =\frac{Y^{v_{A}+v_{B}+n} \mathrm{e}^{-Y}}{\Gamma\left(v_{B}+1\right) \Gamma\left(v_{A}+n\right)}{ }_{1} F_{1}\left(\begin{array}{c}
v_{B}+1 \\
v_{A}+v_{B}+n+1
\end{array} \mid\left(1-\sigma_{k}^{-1}\right) Y\right)
\end{aligned}
$$

where ${ }_{1} F_{1}$ is the confluent hypergeometric function, see for example, [29, formula 13.4.1]. Thus the eigenvalues of $Y=L+X$ have the joint density

$$
\propto \Delta_{n}(y) \operatorname{det}\left[g_{k}\left(y_{j}\right)\right]_{j, k=1}^{n}
$$

which corresponds to [27, formula (15)]. 
16 A. B. J. Kuijlaars et al.

The proof of [27] uses among others on an integral over the unitary group, see [27, formula (13)], that is special to confluent hypergeometric functions. This approach, therefore, does not seem to generalize to the more general setting of Corollary 3.2.

\subsection{Polynomial ensemble of derivative type}

Polynomial ensembles of derivative type were introduced by Kieburg and Kösters in $[22,23]$ in the connection with products of random matrices. There is an analogous notion that is relevant for sums of random matrices, and we call it polynomial ensemble of derivative type (in the additive sense).

The polynomial ensemble (3.9) is of derivative type (in the additive sense) if

$$
\operatorname{span}\left\{w_{1}, \ldots, w_{n}\right\}=\operatorname{span}\left\{w^{(k)} \mid k=0, \ldots, n-1\right\}
$$

for some function $w$ that is at least $n-1$ times differentiable. In that case, we may use elementary column transformations to the determinant in (3.9) to pass from $w_{1}, \ldots, w_{n}$ to $W, W^{\prime}, \ldots, W^{(n-1)}$ (with a possibly different normalization constant). The corresponding probability density (3.10) then is

$$
f(A)=f\left(x_{1}, \ldots, x_{n}\right) \propto \frac{\operatorname{det}\left[W^{(k-1)}\left(x_{j}\right)\right]_{j, k=1}^{n}}{\Delta_{n}(x)}
$$

and the density on eigenvalues is

$$
\frac{1}{Z_{n}} \Delta_{n}(x) \operatorname{det}\left[W^{(k-1)}\left(x_{j}\right)\right]_{j, k=1}^{n}
$$

The spherical transform (3.11) simplifies in this case since

$$
\int_{-\infty}^{\infty} W^{(k-1)}(x) \mathrm{e}^{-i s x} \mathrm{~d} x=(i s)^{k-1} \int_{-\infty}^{\infty} w(x) \mathrm{e}^{-i s x} \mathrm{~d} x
$$

and

$$
\begin{aligned}
\widehat{f}\left(s_{1}, \ldots, s_{n}\right) & \propto \frac{1}{\Delta_{n}(s)} \operatorname{det}\left[\left(i s_{j}\right)^{k-1}\right]_{j, k=1}^{n} \prod_{j=1}^{n} \int_{-\infty}^{\infty} w(x) \mathrm{e}^{-i s_{j} x} \mathrm{~d} x \\
& \propto \prod_{j=1}^{n} \int_{-\infty}^{\infty} w(x) \mathrm{e}^{-i s_{j} x} \mathrm{~d} x .
\end{aligned}
$$


The proportionality constant follows from the property $\widehat{f}(0, \ldots, 0)=1$. Thus for a polynomial ensemble of derivative type (in the additive sense) the spherical transform factorizes as

$$
\widehat{f}\left(s_{1}, \ldots, s_{n}\right)=\frac{1}{\left(\int_{-\infty}^{\infty} W(x) \mathrm{d} x\right)^{n}} \prod_{j=1}^{n}\left(\mathcal{F}_{W}\right)\left(s_{j}\right)
$$

where $\mathcal{F}$ is again the Fourier transform (3.14). The formula (3.19) is similar to [23, Corollary 3.2] that applies to the multiplicative setting where the Mellin transform is used instead of the Fourier transform.

We write $X \sim D P E(W)$ if $X$ is a random Hermitian matrix whose eigenvalues are a polynomial ensemble as in (3.16) with function $w$. The following is now almost immediate. It is the analogue of [23, Corollary 3.4$]$ in the additive setting.

Corollary 3.4. If $X$ and $Y$ are independent unitarily invariant random Hermitian matrices whose eigenvalues are polynomial ensembles of derivative type (in the additive sense), say $X \sim D P E\left(w_{1}\right)$ and $Y \sim D P E\left(w_{2}\right)$, then $X+Y$ is a random matrix whose eigenvalues are a polynomial ensemble of derivative type (in the additive sense)

$$
X+Y \sim D P E\left(w_{1} * w_{2}\right),
$$

where $*$ denotes the usual convolution of functions on the real line

$$
w_{1} * w_{2}(x)=\int_{-\infty}^{\infty} w_{1}(x-y) w_{2}(y) d y
$$

Proof. This follows from Theorem 1.1, (3.19), and the basic properties of the Fourier transform.

Using (3.11) and (3.19) in Theorem 1.1 we obtain the following result.

Corollary 3.5. If $X$ and $Y$ are independent unitarily invariant random Hermitian matrices with the eigenvalues of $X$ a polynomial ensembles of derivative type (in the additive sense) say $X \sim D P E(W)$ and the eigenvalues of $Y$ a polynomial ensemble $Y \sim P E\left(W_{1}, \ldots, w_{n}\right)$, then $X+Y$ is a random matrix whose eigenvalues are a polynomial ensemble

$$
X+Y \sim P E\left(W * w_{1}, \ldots, W * W_{n}\right) .
$$


Proof. This is very similar, and we omit the proof. See [23, Theorem 3.3] for the analogous result in the multiplicative setting.

Remark 3.6. If a function $w$ generates a polynomial ensemble (3.17) then clearly (3.17) should be nonnegative for every choice of $x_{1}, x_{2}, \ldots, x_{n}$. This property is satisfied by so-called Pólya frequency functions, see for example [21], for which it holds that

$$
\operatorname{det}\left[W^{(k-1)}\left(x_{j}\right)\right] \geq 0
$$

whenever $x_{1}<x_{2}<\cdots<x_{n}$. Faraut [15] has an interesting survey that connects Pólya frequency functions to the representation theory of Gelfand pairs, including (U(n) $\ltimes$ $\operatorname{Herm}(n), \mathrm{U}(n))$.

\section{More on Addition with LUE Matrix}

\subsection{Alternative proof of Corollary 3.2}

We give a different proof of Corollary 3.2, based on a change of variables. When integrating out the eigenvectors of $Y$ we encounter a matrix integral over the unitary group that was recently evaluated by Kieburg et al. [24]

Assume first that $X$ is fixed with eigenvalues $x_{1}, \ldots, x_{n}$ and $L$ is an LUE matrix with parameter $\alpha>-1$. Then $L \mapsto Y=X+L$ is a change of variables. From (3.5), we arrive at the probability density

$$
\propto \operatorname{det}(Y-X)^{\alpha} \mathrm{e}^{-\operatorname{Tr}(Y-X)}
$$

on the set of Hermitian matrices $Y$ with $Y \geq X$. Letting $Y_{1}, \ldots, Y_{n}$ be the eigenvalues of $Y$, this is

$$
\propto\left(\prod_{j=1}^{n} \mathrm{e}^{-y_{j}}\right)\left(\prod_{k=1}^{n} \mathrm{e}^{X_{k}}\right) \operatorname{det}(Y-X)^{\alpha} \mathbf{1}_{Y \geq X}
$$

Introduce the eigenvalue decomposition $Y=U D U^{*}$ with a diagonal matrix $D=$ $\operatorname{diag}\left(y_{1}, \ldots, y_{n}\right)$ and a unitary matrix $U$. The Jacobian of the eigenvalue decomposition is proportional to $\Delta_{n}(y)^{2}$, and we obtain from (4.1) for the density of eigenvalues

$$
\propto \Delta_{n}(y)^{2}\left(\prod_{j=1}^{n} \mathrm{e}^{-Y_{j}}\right)\left(\prod_{k=1}^{n} \mathrm{e}^{x_{k}}\right) \int_{U \in \mathrm{U}(n): U D U^{*} \geq X} \operatorname{det}\left(U D U^{*}-X\right)^{\alpha} \mathrm{d} U
$$


with a proportionality constant that does not depend on $X$. The integral over the subset of the unitary group was evaluated in [24, Theorem 2.3] where it was found to be proportional to

$$
\frac{\operatorname{det}\left[\left(y_{j}-x_{k}\right)_{+}^{\alpha+n-1}\right]_{j, k=1}^{n}}{\Delta_{n}(x) \Delta_{n}(y)} .
$$

Here $(y-x)_{+}=\max (y-x, 0)$. The density (4.2) therefore is

$$
\propto \frac{\Delta_{n}(y)}{\Delta_{n}(x)}\left(\prod_{j=1}^{n} e^{-y_{j}}\right)\left(\prod_{k=1}^{n} e^{x_{k}}\right) \operatorname{det}\left[\left(y_{j}-x_{k}\right)_{+}^{\alpha+n-1}\right]_{j, k=1}^{n} .
$$

We bring the prefactors into the determinant and obtain

$$
\propto \frac{\Delta_{n}(y)}{\Delta_{n}(x)} \operatorname{det}\left[\left(y_{j}-x_{k}\right)_{+}^{\alpha+n-1} \mathrm{e}^{-y_{j}+x_{k}}\right]_{j, k=1}^{n} .
$$

This is the result for a fixed matrix $X$ and (4.4) shows that the eigenvalues of $Y=X+L$ are a polynomial ensemble.

Now suppose that $X$ is random, independent of $L$, and its eigenvalues are a polynomial ensemble (3.9). Then by the Andreief formula (3.1), we find from (4.4) after averaging over (3.9), that the eigenvalues of $Y=X+L$ have joint density

$$
\propto \Delta_{n}(y) \operatorname{det}\left[\int_{-\infty}^{\infty}\left(y_{j}-x\right)_{+}^{\alpha+n-1} \mathrm{e}^{-y_{j}+x} f_{k}(x) \mathrm{d} x\right]_{j, k=1}^{n} .
$$

We change variables $x \mapsto y_{j}-x$ and arrive at

$$
\propto \Delta_{n}(y) \operatorname{det}\left[\int_{0}^{\infty} x^{\alpha+n-1} \mathrm{e}^{-x} f_{k}\left(y_{j}-x\right) \mathrm{d} x\right]_{j, k=1}^{n}
$$

which is indeed a polynomial ensemble with the functions (3.15) as claimed in Corollary 3.2 .

\subsection{Biorthogonal functions}

Corollary 3.2 is a transformation result for polynomial ensembles. A polynomial ensemble is a special case of a determinantal point process. The correlation kernel for a polynomial ensemble

$$
\frac{1}{Z_{n}} \Delta_{n}(x) \operatorname{det}\left[f_{j}\left(x_{k}\right)\right]_{j, k=1}^{n}
$$


takes the form

$$
\sum_{k=0}^{n-1} p_{k}(x) q_{k}(y)
$$

where each $p_{k}$ is a monic polynomial of degree $k$, each $q_{k}$ belongs to the linear span of $f_{1}, \ldots, f_{n}$ and the biorthogonality condition

$$
\int_{-\infty}^{\infty} p_{j}(x) q_{k}(x) \mathrm{d} x=\delta_{j, k}
$$

holds, see [9, 17].

Suppose $Y=X+L$ where $X \sim P E\left(f_{1}, \ldots, f_{n}\right)$ and $Y \sim P E\left(g_{1}, \ldots, g_{n}\right)$ as in Corollary 3.2. We write $K_{n}^{X}$ and $K_{n}^{Y}$ for the correlation kernels of the two polynomial ensembles and

$$
\begin{aligned}
& K_{n}^{X}(x, y)=\sum_{k=0}^{n-1} p_{k}(x) q_{k}(y) \\
& K_{n}^{Y}(x, y)=\sum_{k=0}^{n-1} P_{k}(x) Q_{k}(y),
\end{aligned}
$$

and we investigate the relation between the two sets of biorthogonal functions. The transformation results are in formulas (4.9), (4.18), and (4.19) below. See [12] for similar results related to addition with a GUE matrix.

We assume $p_{k}$ and $q_{k}$ are given. We fix $Q_{k}$ by taking

$$
Q_{k}(y)=\frac{1}{\Gamma(\alpha+n)} \int_{0}^{\infty} x^{\alpha+n-1} \mathrm{e}^{-x} q_{k}(y-x) \mathrm{d} x, \quad k=0, \ldots, n-1 .
$$

Then the $Q_{k}$ are in the linear span of $g_{1}, \ldots, g_{n}$ because of (3.15) and the fact that each $q_{k}$ is in the linear span of $f_{1}, \ldots, f_{n}$. We want to find polynomials $P_{0}, \ldots, P_{n-1}$ such that

$$
\int_{-\infty}^{\infty} P_{j}(x) Q_{k}(x) \mathrm{d} x=\delta_{j, k}
$$

and $\operatorname{deg} P_{j}=j$ for $j=0, \ldots, n-1$.

We calculate from (4.9) (for an as yet unknown $P_{j}$ )

$$
\int_{-\infty}^{\infty} P_{j}(x) Q_{k}(x) \mathrm{d} x=\frac{1}{\Gamma(\alpha+n)} \int_{-\infty}^{\infty} \int_{0}^{\infty} t^{\alpha+n-1} \mathrm{e}^{-t} P_{j}(x) q_{k}(x-t) \mathrm{d} t \mathrm{~d} x
$$

and make the changes of variables $t^{\prime}=x-t, s^{\prime}=t$. Then by Fubini's theorem

$$
\int_{-\infty}^{\infty} P_{j}(x) Q_{k}(x) \mathrm{d} x=\frac{1}{\Gamma(\alpha+n)} \int_{-\infty}^{\infty}\left(\int_{0}^{\infty} s^{\alpha+n-1} \mathrm{e}^{-s} P_{j}(t+s) \mathrm{d} s\right) q_{k}(t) \mathrm{d} t .
$$


We want to choose $P_{k}$ such that

$$
\frac{1}{\Gamma(\alpha+n)} \int_{0}^{\infty} s^{\alpha+n-1} \mathrm{e}^{-s} P_{k}(x+s) \mathrm{d} s=p_{k}(x)
$$

for $k=0,1, \ldots, n-1$, since then by (4.10) and the biorthogonality for the $p_{j}{ }^{\prime} \mathrm{s}$ and $q_{k}{ }^{\prime} \mathrm{s}$,

$$
\int_{-\infty}^{\infty} P_{j}(x) Q_{k}(x) \mathrm{d} x=\int_{-\infty}^{\infty} p_{j}(t) q_{k}(t) \mathrm{d} t=\delta_{j, k} .
$$

The mapping $\mathcal{L}: f \mapsto \mathcal{L} f$ with

$$
\mathcal{L} f(x)=\frac{1}{\Gamma(\alpha+n)} \int_{0}^{\infty} s^{\alpha+n-1} \mathrm{e}^{-s} f(x+s) \mathrm{d} s
$$

maps polynomials to polynomials of the same degree and the same leading coefficient. Consider the polynomials $e_{k}(x)=\frac{1}{k !} x^{k}$. We have by (4.12) and the binomial theorem

$$
\begin{aligned}
\mathcal{L} e_{k}(x) & =\frac{1}{k ! \Gamma(\alpha+n)} \int_{0}^{\infty} s^{\alpha+n-1} \mathrm{e}^{-s}(x+s)^{k} \mathrm{~d} s \\
& =\sum_{j=0}^{k} \frac{1}{j !(k-j) ! \Gamma(\alpha+n)} \int_{0}^{\infty} s^{\alpha+n-1} \mathrm{e}^{-s} x^{k-j} s^{j} \mathrm{~d} s \\
& =\sum_{j=0}^{k} \frac{\Gamma(\alpha+n+j)}{j !(k-j) ! \Gamma(\alpha+n)} x^{k-j} \\
& =\sum_{j=0}^{k}\left(\begin{array}{c}
\alpha+n+j-1 \\
j
\end{array}\right) e_{k-j}(x) .
\end{aligned}
$$

Since $e_{k-j}=e_{k}^{(j)}$, we conclude that

$$
\mathcal{L} f=\sum_{j=0}^{\infty}\left(\begin{array}{c}
\alpha+n+j-1 \\
j
\end{array}\right) f^{(j)}
$$

if $f$ is one of the functions $e_{k}$, and then by linearity for arbitrary polynomials $f$. Note that (4.13) is a finite sum if $f$ is a polynomial.

To invert $\mathcal{L}$ we need a sequence $\left(a_{k}\right)_{k}$ such that $a_{0}=1$ and

$$
\sum_{j=0}^{k} a_{k-j}\left(\begin{array}{c}
\alpha+n+j-1 \\
j
\end{array}\right)=0, \quad k=1,2, \ldots,
$$

since then we can put

$$
\mathcal{L}^{-1} f=\sum_{k=0}^{\infty} a_{k} f^{(k)}
$$


22 A. B. J. Kuijlaars et al.

The recurrence (4.14) is solved by the numbers

$$
a_{k}=(-1)^{k}\left(\begin{array}{c}
\alpha+n \\
k
\end{array}\right)
$$

Indeed, note that

$$
(-1)^{j}\left(\begin{array}{c}
\alpha+n+j-1 \\
j
\end{array}\right)=\left(\begin{array}{c}
-\alpha-n \\
j
\end{array}\right)
$$

and then

$$
\begin{aligned}
\sum_{j=0}^{k}(-1)^{k-j}\left(\begin{array}{c}
\alpha+n \\
k-j
\end{array}\right)\left(\begin{array}{c}
\alpha+n+j-1 \\
j
\end{array}\right) & =(-1)^{k} \sum_{j=0}^{k}\left(\begin{array}{c}
\alpha+n \\
k-j
\end{array}\right)\left(\begin{array}{c}
-\alpha-n \\
j
\end{array}\right) \\
& =(-1)^{k}\left(\begin{array}{l}
0 \\
k
\end{array}\right)
\end{aligned}
$$

by the Chu-Vandermonde identity.

The conclusion from (4.12), (4.15), and (4.16) is that

$$
P_{k}(x)=\sum_{j=0}^{k}(-1)^{j}\left(\begin{array}{c}
\alpha+n \\
j
\end{array}\right) p_{k}^{(j)}(x)
$$

is indeed a monic polynomial of degree $k$ that satisfies (4.11) and the biorthogonality property

$$
\int_{-\infty}^{\infty} P_{j}(x) Q_{k}(x) \mathrm{d} x=\delta_{j, k}, \quad j, k=0, \ldots, n-1
$$

holds.

There is a contour integral formula for $P_{k}$

$$
P_{k}(x)=\frac{\Gamma(\alpha+n+1)}{2 \pi i} \int_{C} \frac{p_{k}(x-s)}{s^{\alpha+n+1}} \mathrm{e}^{s} \mathrm{~d} s
$$

where $C$ is a contour encircling the negative real axis, starting at $-\infty$ in the lower half plane, and ending at $-\infty$ in the upper half plane. [It can be taken to be a closed contour around the origin if $\alpha$ is an integer.] To prove the integral formula (4.18) we define a mapping $\mathcal{M}$ by

$$
\mathcal{M} f(x)=\frac{\Gamma(\alpha+n+1)}{2 \pi i} \int_{C} \frac{f(x-s)}{s^{\alpha+n+1}} \mathrm{e}^{s} \mathrm{~d} s
$$


and we evaluate $\mathcal{M} e_{k}$ where $e_{k}(x)=\frac{1}{k !} x^{k}$ as before. By the binomial theorem

$$
\mathcal{M} e_{k}=\frac{\Gamma(\alpha+n+1)}{2 \pi i k !} \int_{C} \sum_{j=0}^{k}(-1)^{j} X^{k-j}\left(\begin{array}{l}
k \\
j
\end{array}\right) \frac{1}{s^{\alpha+n-j+1}} \mathrm{e}^{s} \mathrm{~d} s
$$

which is

$$
\sum_{j=0}^{k} \frac{(-1)^{j}}{j !} e_{k-j} \frac{\Gamma(\alpha+n+1)}{\Gamma(\alpha+n-j+1)}
$$

and this is

$$
\sum_{j=0}^{k}(-1)^{j}\left(\begin{array}{c}
\alpha+n \\
j
\end{array}\right) e_{k}^{(j)}
$$

By linearity we have

$$
\mathcal{M} f(x)=\sum_{j=0}^{\infty}(-1)^{j}\left(\begin{array}{c}
\alpha+n \\
j
\end{array}\right) f^{(j)}
$$

for every polynomial $f$, which indeed coincides with the formula (4.15)-(4.16) for $\mathcal{L}^{-1}$.

For the correlation kernels we arrive at the transformation formula

$$
\begin{aligned}
K_{n}^{Y}(x, y) & =\sum_{k=0}^{n-1} P_{k}(x) Q_{k}(y) \\
& =\frac{\alpha+n}{2 \pi i} \int_{C} \int_{0}^{\infty}\left(\frac{t}{s}\right)^{\alpha+n} \mathrm{e}^{s-t} K_{n}^{X}(x-s, y-t) \frac{\mathrm{d} t}{t} \frac{\mathrm{d} s}{s},
\end{aligned}
$$

which could be useful for asymptotic analysis. A similar formula for the case of a sum with a GUE matrix was given in [12] and it was used for asymptotic analysis in [10] and [11].

\section{Funding}

A.K. is supported by long-term structural funding-Methusalem grant of the Flemish Government, the Belgian Interuniversity Attraction Pole P07/18, KU Leuven Research Grant OT/12/073, and FWO Flanders projects G.0934.13 and G.0864.16. The research of P.R. is supported by the Radboud Excellence Fellowship. P.R. was partially supported by CONICET grant PIP 112-200801-01533, FONCYT grant PICT 2014-3452, and by SECyT-UNC. 


\section{Acknowledgements}

The first author is very grateful to Mario Kieburg and Holger Kösters for inspiring discussions related to their papers [22, 23]. We thank Peter Forrester and Tom Koornwinder for their useful remarks and pointers to the literature. We also thank Erik Koelink for helpful discussions. We are very grateful to the anonymous referees for helpful remarks and for pointing out a number of inaccuracies in the first version of this article.

\section{References}

[1] Adhikari, K., N. Reddy, T. Reddy, and K. Saha. "Determinantal point processes in the plane from products of random matrices." Ann. Inst. Henri Poincaré Probab. Stat. 52 (2016): 16-46.

[2] Akemann, G. and Z. Burda. "Universal microscopic correlation functions for products of independent Ginibre matrices." J. Phys. A 45, no. 46 (2012): 465201, 18 pp.

[3] Akemann, G., Z. Burda, M. Kieburg, and T. Nagao. "Universal microscopic correlation functions for products of truncated unitary matrices." J. Phys. A. 47, no. 25 (2014): 255202, $26 \mathrm{pp}$.

[4] Akemann, G. and J. Ipsen. "Recent exact and asymptotic results for products of independent random matrices." Acta Phys. Polon. B 46, no. 9 (2015): 1747-84.

[5] Akemann, G., J. Ipsen, and M. Kieburg. "Products of rectangular random matrices: singular values and progressive scattering." Phys. Rev. E 88 052118, (2013): 13 pp.

[6] Akemann, G., M. Kieburg and L. Wei. "Singular value correlation functions for products of Wishart random matrices." J. Phys. A 46, no. 27, (2013): 275205, 22 pp.

[7] Ben Saïd, S. and B. Ørsted. "Analysis on flat symmetric spaces." J. Math. Pures Appl. 84, no. 10 (2005): pp 1393-426.

[8] Benson, C., J. Jenkins, and G. Ratcliff. "On Gelfand pairs associated with solvable Lie groups." Trans. Amer. Math. Soc. 321, no. 1 (1990): 85-116.

[9] Borodin, A. “Biorthogonal ensembles." Nuclear Phys. B 536 (1998): 704-32.

[10] Claeys, T. and A. Doeraene. "Gaussian perturbations of hard edge random matrix ensembles." Nonlinearity 29 (2016): 3385-416.

[11] Claeys, T., A. B. J. Kuijlaars, K. Liechty, and D. Wang. "Propagation of singular behavior for Gaussian perturbations of random matrices." arxiv:1608.05870.

[12] Claeys, T., A. B. J. Kuijlaars, and D. Wang. "Correlation kernels for sums and products of random matrices." Random Matrices Theory Appl. 4 (2015): 155017, 31 pp.

[13] Deift, P. and D. Gioev. Random Matrix Theory: Invariant Ensembles and Universality. Courant Lecture Notes in Mathematics 18. Providence RI: Amer. Math. Soc., 2009.

[14] van Dijk, G. Introduction to Harmonic Analysis and Generalized Gelfand Pairs. De Gruyter Studies in Mathematics 36. Berlin: Walter de Gruyter \& Co, 2009.

[15] Faraut, J. "Infinite Dimensional Harmonic Analysis and Probability." In Probability Measures on Groups: Recent Directions and Trends, edited by S. G. Dani and P. Graczyk, 179-254. Mumbai: Tata Inst. Fund. Res., 2006. 
[16] Faraut, J. “Asymptotic spherical analysis on the Heisenberg group." Colloq. Math. 118, no. 1 (2010): 233-58.

[17] Forrester, P. J. Log-gases and Random Matrices. Princeton NJ: Princeton University Press, 2010.

[18] Forrester, P. J. and E. Rains. "Interpretations of some parameter dependent generalizations of classical matrix ensembles." Probab. Theory Related Fields 131 (2005): 1-61.

[19] Gelfand, I. M. and M. A. Naimark. Unitäre Darstellungen der klassischen Gruppen (German). Berlin: Akademie, 1957.

[20] Helgason, S. Groups and Geometric Analysis. Integral Geometry, Invariant Differential Operators, and Spherical Functions. Pure and Applied Mathematics 113. Orlando, FL: Academic Press, 1984.

[21] Karlin, S. Total Positivity, vol. I. Stanford, CA: Stanford University Press, 1968.

[22] Kieburg, M. and H. Kösters. "Exact relation between singular value and eigenvalue statistics." Random Matrices Theory Appl. 5 (2016): 1650015, 57 pp.

[23] Kieburg, M. and H. Kösters. "Products of random matrices from polynomial ensembles." arXiv:1601.03724.

[24] Kieburg, M., A. B. J. Kuijlaars, and D. Stivigny. "Singular value statistics of matrix products with truncated unitary matrices." Int. Math. Res. Not. IMRN 2016, no. 11, (2016): pp. 3392-424.

[25] Kuijlaars, A. B. J. "Transformations of Polynomial Ensembles." In Modern Trends in Constructive Function Theory, edited by D. P. Hardin, D. S. Lubinsky and B. Simanek, 253-68. Contemp. Math. 661, Providence RI: American Mathematical Society, 2016.

[26] Kuijlaars, A. B. J. and D. Stivigny. "Singular values of products of random matrices and polynomial ensembles." Random Matrices Theory Appl. 3 (2014): 1450011, 22 pp.

[27] Kumar, S. "Eigenvalue statistics for the sum of two complex Wishart matrices." EuroPhys. Lett. 107 (2014): 60002.

[28] Kumar, S. "Random matrix ensembles involving Gaussian Wigner and Wishart matrices, and biorthogonal structure." Phys. Rev. E 92 (2015): 032903.

[29] Olver, F. W. J., D. W. Lozier, R. F. Boisvert, and C. W. Clark, eds. NIST Handbook of Mathematical Functions. Cambridge University Press, Cambridge, 2010. 